\title{
BMJ Open Cross-sectional study protocol for the Arabic Healthy Weight Project promoting active living and healthy eating among Arabic-speaking communities in South Western Sydney
}

Vilas Kovai, ${ }^{\oplus 1}$ Punitha Arjunan, ${ }^{1}$ Danielle Weber, ${ }^{1}$ Janelle Rooney, ${ }^{1}$ Bin Jalaludin, ${ }^{2}$ Karen Wardle, ${ }^{1}$ Mandy Williams ${ }^{1}$

To cite: Kovai $\mathrm{V}$, Arjunan $\mathrm{P}$, Weber $\mathrm{D}$, et al. Cross-sectional study protocol for the Arabic Healthy Weight Project promoting active living and healthy eating among Arabicspeaking communities in South Western Sydney. BMJ Open 2019;9:e025502. doi:10.1136/ bmjopen-2018-025502

- Prepublication history for this paper is available online. To view these files, please visit the journal online (http://dx.doi org/10.1136/bmjopen-2018025502).

Received 19 July 2018 Revised 7 February 2019 Accepted 12 February 2019

Check for updates

(c) Author(s) (or their employer(s)) 2019. Re-use permitted under CC BY-NC. No commercial re-use. See rights and permissions. Published by BMJ.

${ }^{1}$ Population Health/Health Promotion Service, South Western Sydney Local Health District, Liverpool, New South Wales, Australia

${ }^{2}$ Population Health Intelligence, Healthy People and Places Unit, South Western Sydney Local Health District, Liverpool, New South Wales, Australia

Correspondence to

Dr Vilas Kovai;

Vilas.Kovai@health.nsw.gov.au

\section{ABSTRACT}

Introduction The South Western Sydney Local Health District (SWSLHD) is home to nearly 75000 Arabicspeaking people. Of these, nearly three quarters are overweight or obese and suffer from a range of chronic diseases. To address this, the Health Promotion Service of SWSLHD will conduct a community-based overweight and obesity prevention intervention (Arabic Healthy Weight Project, 2018-2021) with Arabic community members aged between 18 and 50 years. The intervention's main activities will include a comprehensive social marketing campaign and an 'Eat-Move-Live Healthy' programme. Methods and analysis The project will be evaluated using a pre-postintervention study design to measure changes in practices in relation to physical activity, consumption of vegetables and intake of sugar-sweetened beverages. The evaluation will apply mixed data collection methods. The quantitative data will be collected using a face-to-face survey of 1540 participants from two independent samples (pre: 770 and post: 770 ). Descriptive and inferential statistical tests will be used to analyse the quantitative data. The qualitative component will use focus group discussions and interviews to evaluate the formative, process and follow-up phases of data collection. A combination of deductive and inductive methods of data analysis will be conducted using NVivo software.

Ethics and dissemination The protocol has been approved by the Human Research Ethics Committee of SWSLHD (HREC/16/LPOOL/303). Findings will be published in peer-reviewed journals.

\section{INTRODUCTION}

The state of New South Wales (NSW) in Australia is home to many culturally and linguistically diverse (CALD) communities. ${ }^{1}$ Over one quarter of NSW residents (27.6\%) were born overseas and $31 \%$ speak a language other than English at home. Arabic is the third most commonly spoken language in NSW. ${ }^{1}$ South Western Sydney Local Health District (SWSLHD) mirrors this diversity, ${ }^{2}$ with Arabic-speaking communities,
Strengths and limitations of this study

- First population-based study to promote active living and healthy eating among Arabic-speaking communities in Australia.

- If proven effective, the project will provide a useful model for culturally and linguistically diverse community interventions targeting healthy weight.

- No causal inferences can be made as the sample is not randomised, and a control group will not be used to compare groups or assess the effects of the intervention.

predominately from a Middle-Eastern background, making up the main ethnic group, and with the majority (38643 or $52 \%$ of the total Arabic-speaking communities in SWSLHD) residing in the Bankstown area. ${ }^{2}$

Arabic-speaking people, like their Australian-born counterparts, suffer from a range of chronic diseases, due in part to unhealthy lifestyles. $^{3} 4$ Regular physical activity can contribute to better health outcomes and reduce obesity levels and the risk of chronic disease ${ }^{56} \mathrm{~A}$ diet high in vegetables can also contribute to improved health. ${ }^{7}$ Only $36.9 \%$ of Lebanese-born people (the main Arabicspeaking group in Australia), however, meet the recommended levels of physical activity, while just $2 \%$ meet the recommended five serves of vegetables per day. ${ }^{4}$

It would seem that physical inactivity is common across most migrant CALD groups for reasons associated with their cultural and religious beliefs, as well as the socioeconomic challenges and environmental barriers ${ }^{8-10}$ they experience. Barriers to physical activity include: a lack of time, reliance on an exercise companion, family responsibilities and cost. ${ }^{11}$ Although they figure prominently in 
a traditional diet, vegetables are now widely perceived as expensive, time consuming to prepare and far less a priority than meat.

Recent evidence ${ }^{3}$ suggests that sugar-sweetened beverages (SSBs) are highly consumed within Australia's Arabicspeaking communities. Moreover, there is strong evidence of a direct relationship between SSB intake and overweight and obesity, which are, in turn, strongly linked to chronic conditions such as cardiovascular disease and diabetes. ${ }^{12} 13$

The literature suggests that interventions are more likely to be effective ${ }^{14}{ }^{15}$ if they are culturally relevant and tailored, ${ }^{891617}$ use existing social structures ${ }^{81518}$ and involve respected role models. Moreover, interventions also need to be multistrategy and collaborative to address multiple levels of influence, including individual, cultural/societal and environmental factors.

Obesity prevention interventions in the general population also have the potential to be culturally modified for CALD communities. Social marketing approaches, for example, have been effective in increasing physical activity in adults, reducing SSBs and fast-food consumption at home ${ }^{12}$ and changing attitudes and behaviours, particularly when part of a multistrategy approach. ${ }^{12} 1920$

Community-based physical activity classes are considered "promising ${ }^{17}$ when offering free fitness instruction and aerobics in public places (parks, community centres, sports facilities). Walkable urban design in local areas also shows potential, but needs to be rigorously evaluated. ${ }^{121}$ Other interventions, such as 'point of purchase' strategies, including shelf labels, posters and in-store handouts, have modestly impacted consumption of healthy food. ${ }^{22}$ Evidence also suggests that peer nutrition education can improve knowledge and dietary intake in Latino populations ${ }^{22}$ which benefits may be transferable to other CALD communities.

Given the lack of quality interventions to reduce overweight and obesity in the Arabic-speaking communities, there is a need for well-evaluated population-based initiatives to contribute to the evidence. To address this, the Health Promotion Service of SWSLHD will conduct a community-based overweight and obesity prevention intervention (Arabic Healthy Weight Project [AHWP], 2018-2021) with Arabic community members aged between 18 and 50 years living in Bankstown and surrounding areas. This age group was selected due to higher rates of obesity/overweight in this age group ${ }^{23}$ as well as the likelihood of them influencing their family/ children in adopting healthy lifestyles. In this paper, the term 'project' is used to denote the implementation and effectiveness of a multistrategy intervention. This evaluation will apply mixed data collection methods. The quantitative part will be evaluated using a pre and post design to measure changes in practices in relation to physical activity per week, consumption of vegetables and daily SSB intake. The qualitative component will consist of formative, process and follow-up phases of data collection, designed to provide insight into the success of the AHWP programme in achieving its goals.

\section{Intervention: theoretical framework}

The over-riding theoretical frameworks for the project strategies are derived from the Anderson model of health utilisation, ${ }^{24}$ the Social Cognitive Theory (SCT),${ }^{24}$ and the Transtheoretical Model (TTM) or Stage of Change theory. ${ }^{24}$ Dietary and physical activity behaviour change is associated with a range of factors, including predisposing factors (individual beliefs and attitudes towards the behaviour) and enabling factors (knowledge, skills, observation of others, supportive norms, social support and supportive environment). ${ }^{25-28}$ Furthermore, people move through a series of stages in behaviour change as postulated by TTM. In addition, evidence from the SCT, which also focuses on the environment, suggests that there is a constant interaction between the individual, their social and physical environment and their behaviour. Our intervention strategies are designed accordingly.

\section{Intervention description}

The intervention will engage role models, use existing social structures, including schools and religious organisations, and be guided by a combination of theories. Planned activities include: delivering campaign messages through social marketing (population level); engaging communities through a grants programme that aims to promote nutritional awareness and encourage physical activity (cultural/societal level); enhancing point of sale/ purchase promotions through supermarkets and green grocers (environmental level) and engaging adults in an 'Eat-Move-Live Healthy' programme (healthy lifestyle programme) through organisations/schools/religious institutions to provide facilities, promote the programme and recruit participants (individual level). Table 1 outlines the strategies, how they will be implemented, how long they will run for and expected outcomes.

\section{METHODS AND ANALYSIS}

This section details the methodology underlying the quantitative and qualitative components. This preintervention and postintervention design consists of two quantitative evaluation phases (baseline and follow-up) and three qualitative evaluation phases (formative, process and follow-up).

\section{Quantitative component}

\section{Research and evaluation context}

We accept that changes in obesity rates are unlikely to be achieved during the project period (2018-2021); we anticipate, however, that the impact of multistrategy and multilevel project activities may well be reflected in changes in the rates of overweight by the follow-up phase of the project.

We propose that by increasing levels of physical activity (frequency and duration) to the recommended level; by consuming the required number of servings of vegetable and by decreasing the volume and frequency of SSB intake the number of overweight participants will 


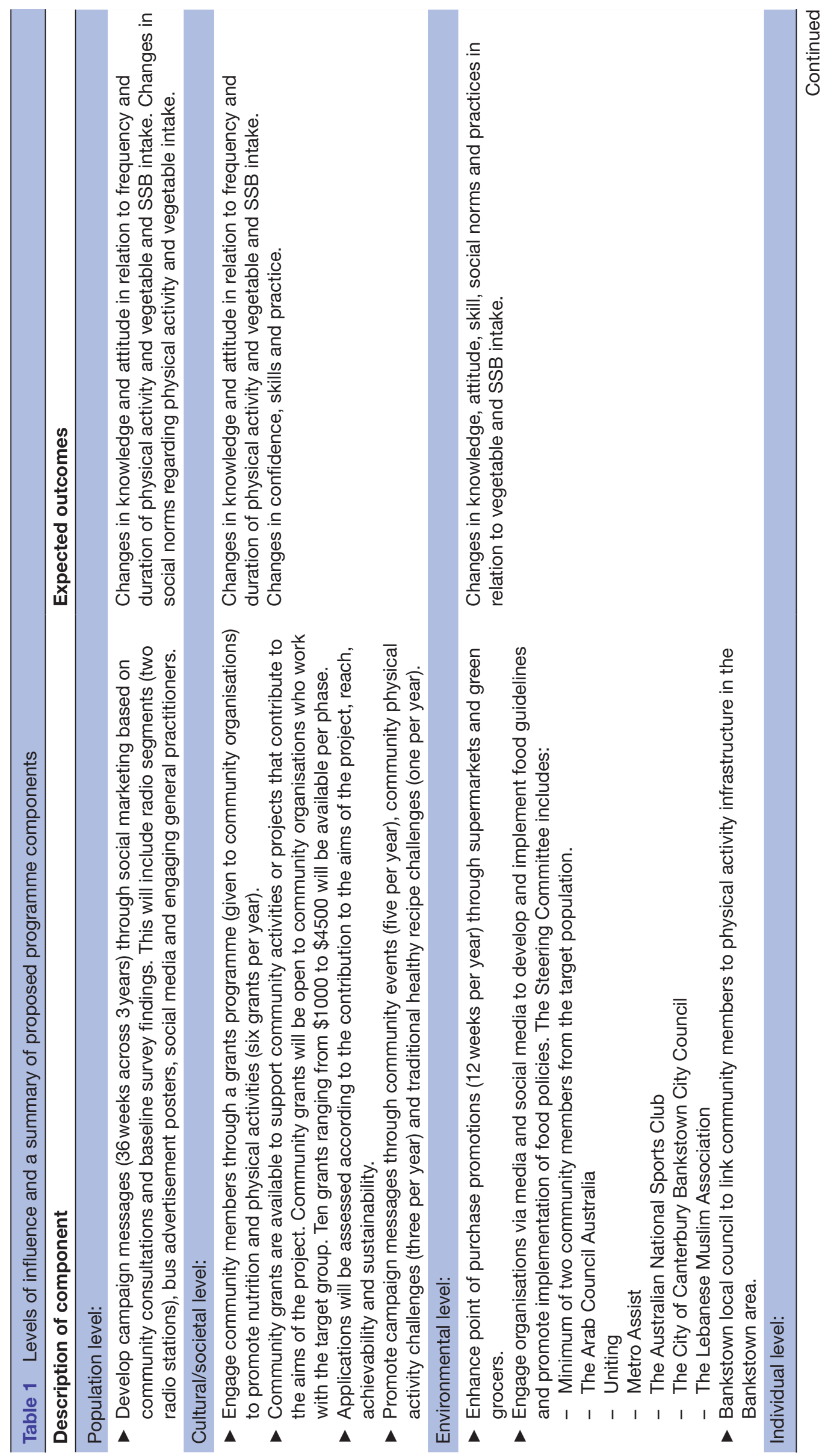


also decrease. In the longer term, this should lead to an overall reduction in participants' body mass index (BMI).

Our literature review revealed that only $37 \%$ of people with Lebanese background meet recommended levels of physical activity. ${ }^{7}$ This project intends, therefore, to effect an incremental increase in physical activity levels by approximately $2.5 \%$ per annum and a total of $7 \%$ increase in the proportion of people undertaking recommended levels of walking and/or vigorous physical activity (per week) over the life of the intervention. This target is in line with the NSW state's aim to reduce overall rates of overweight and obesity by $5 \%$ by $2020 .{ }^{7}$ The clinical significance of such an achievement is supported by the fact that a weight loss of as little as $5 \%-10 \%$ can help to lower blood glucose levels and reduce the risk of developing type 2 diabetes. ${ }^{25}$

In terms of diabetes prevention, there is evidence that increased physical activity, from low levels to up to 1 hour of walking a day, reduce risk. ${ }^{26}$ Likewise, a meta-analysis study posited a link between SSB intake, weight gain and health problems such as diabetes. ${ }^{27}$ Vegetable intake, on the other hand, is inversely associated with cardiovascular disease (CVD) and heart disease, ${ }^{28}$ with 5-6 servings per day is widely believed to reduce CVD and other chronic disease risk. ${ }^{28}$ Hence, the evaluation target of a $7 \%$ change in primary and other outcome measures will allow demonstration of clinical benefit.

The analysis of physical activity trends in the state of NSW, Australia shows that $7 \%$ increase in the proportion of people achieving sufficient level of physical activity over a 3-year period is possible. ${ }^{29}$ Guided by this evidence, the authors believe that if the project activities are implemented as planned, a total of $7 \%$ absolute increase in the proportion of people that will undertake recommended levels of walking and/orvigorous physical activity (per week) over the life of the intervention could be achievable. In addition, the target population is also expected to change their practices with respect to SSB consumption and intake of vegetables to recommended levels in order to achieve a change in health outcomes. While an absolute change of $7 \%$ (from baseline) in reduction of SSBs and increase in vegetable intake is a small change, nevertheless, a small shift in the distribution will lead to a large impact at the population level. ${ }^{30}$

Consultation with project partners and community members confirmed that promoting the practice of taking a regular walk' was a simple and effective means of addressing the issue of physical inactivity among Arabic speakers. Since, however, the concept of 'moderate physical activity' does not easily translate into Arabic idiom, authors of this study pretested the validated questions on 'walking and vigorous physical activity' to ensure more accurate measurement of the primary outcome of this study. 


\section{Objectives}

Primary objective

1. To determine whether the project has increased the proportion of people walking and/or engaging in vigorous physical activity (per week) by $7 \%$ from baseline levels.

\section{Secondary objectives}

1. To determine whether the project has increased the mean frequency and duration of walking and/or vigorous physical activity (per week) among the participants by $7 \%$ from baseline levels.

2. To determine whether the project has decreased the mean volume of SSBs consumed (per day) among the participants by $7 \%$ from baseline levels.

3. To determine whether the project has increased the mean serves of vegetables consumed (per day) by $7 \%$ from baseline levels.

4. To determine the accuracy of self-reported BMI data.

\section{Research questions}

1. To what extent has there been an improvement in weight-related behaviours among adults with Arabic background aged 18-50 years living in Bankstown and surrounding areas of SWSLHD between 2018 and 2021 with respect to:

- Increased walking and vigorous physical activity practices.

- Reduced mean volume intake of SSBs.

- Increased mean serves of vegetables.

- Changes in overweight and obesity rates.

2. To what extent did the project impact the knowledge and 'stages of change in behaviours' (intention, confidence, practice and motivation) leading to improved weight-related behaviours among adults aged 18-50 years, from an Arabic background and living in Bankstown and surrounding areas of SWSLHD between 2018 and 2021?

\section{Evaluation design}

This before and after evaluation aims to measure the outcomes of the AHWP (by 2021). The primary outcome measure is to detect $7 \%$ change in the proportion of people meeting recommended levels of walking and/ or vigorous physical activity. An online baseline survey $(n=770)$ and a follow-up online survey $(n=770)$ will be conducted during the project period. The participants will be recruited using convenient sampling methods, and provide verbal, rather than the usual written, consent, since this deemed to be more culturally appropriate and ethical. $^{31}$ The online survey will be administered face-toface by trained bilingual interviewers using an electronic hand held device (see table 2).

\section{Definitions of outcome measures}

In this study, the term 'physical activity' refers to walking and vigorous physical activity. The secondary outcomes include changes in the proportion of people meeting recommended levels of vegetable and SSB consumption.
Physical activity knowledge

Participants' knowledge of the physical activity guidelines, particularly recommended frequency and duration, will be measured with two pretested (though not validated) questions: 'how many times do you think you should do physical activity in a week?' and 'how many minutes in total do you think you should do physical activity in a week?'

\section{Physical activity: walking and vigorous practices}

Participants will be asked two validated and pretested walking practices questions-in the past week, how many times and for how many minutes in total, have you walked continuously for at least $10 \mathrm{~min}$, whether recreation, physical activity, walking at home (in the garden or around the house) or to get places. ${ }^{32} 33$ A participant involved in $\geq 150 \mathrm{~min}$ of walking accumulated across most days of the week will be considered as having met the recommended level.

Participants will be also asked two validated and pretested vigorous activity questions-in the last week, how many times and how many minutes did you do any vigorous activity which made you breathe harder or puff and pant (eg, gardening, jogging, cycling, aerobics, tennis, gym work, dancing, swimming etc). ${ }^{32} 33$ A participant involved in vigorous physical activity for $\geq 75 \mathrm{~min}$ per week (accumulated on most days of the week) will be considered as having met the recommended physical activity levels. $^{34}$

\section{Height and weight measurement}

To the best of our knowledge, the accuracy of self-reported BMI has not been reported for CALD groups in Australia, such as Arabic speakers. Therefore, both self-reported and measured height and weight data will be collected to assess the validity between these measurements and the extent of self-report bias in this project. The self-reported body height and weight will be collected as part of the interview and height and weight measurements will be taken by a trained researcher immediately following the interview. Height will be measured without shoes, with the respondent looking straight ahead, heels together and feet at an angle of $45^{\circ}$, with a wall mounted stadiometer to the nearest $0.1 \mathrm{~cm}$. Weight will be measured to the nearest $0.1 \mathrm{~kg}$ on calibrated digital scales. BMI will be computed as weight divided by squared height in $\mathrm{kg} / \mathrm{m}^{2}$. Overweight is defined as BMI $\geq 25.0-29.9 \mathrm{~kg} / \mathrm{m}^{2}$ and obesity defined as $\mathrm{BMI} \geq 30.0 \mathrm{~kg} / \mathrm{m}^{2}$, according to WHO criteria. $^{35}$

\section{Recommended serves of vegetables}

One serving of vegetables is defined as half a cup of cooked vegetables or one cup of raw or salad vegetables. ${ }^{36}$ A participant who consumes five serves of vegetables per day will be seen to have met recommended dietary requirements. ${ }^{36}$

\section{Consumption of SSB}

Data will be collected on three types of SSBs (excluding diet drinks) consumed in cups per day (one cup=250 mL). 


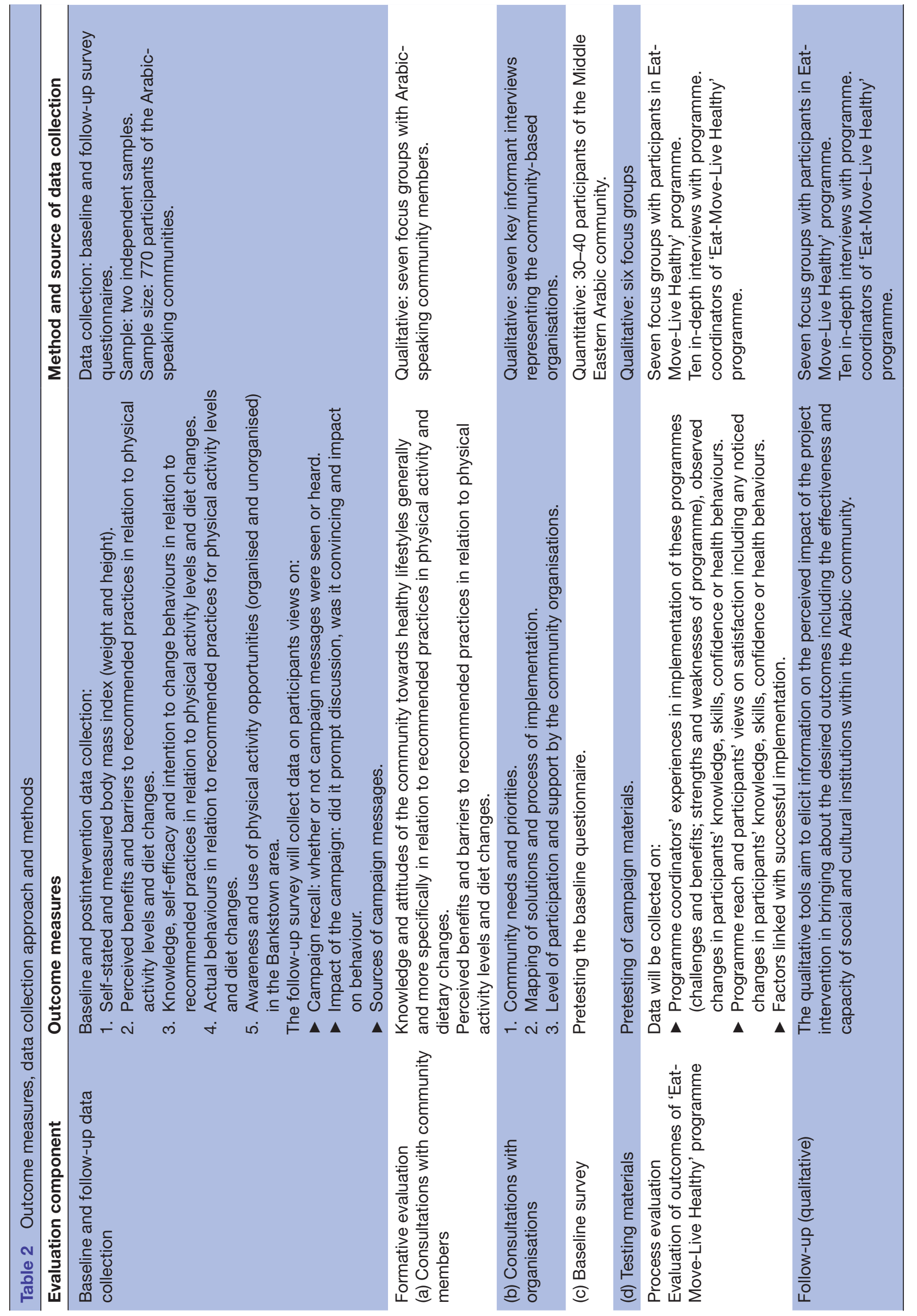


Participants will be shown the models of drink sizes to elicit the correct information and be asked- ${ }^{1}$ how many cups of soft drink, cordial or sports drinks, such as lemonade or Gatorade, do you usually drink in a day? ${ }^{2}$ how many cups of fruit juice or fruit juice drink do you usually drink in a day? and ${ }^{3}$ how many cups of sweetened black tea (with sugar) do you usually drink in a day? Apart from the last, these questions were validated and pretested. ${ }^{32}$

\section{Stages of change in relation to recommended physical activity and vegetable intake}

Participants will be stratified into two stages of change using a two-step algorithm. ${ }^{37}$ In step 1 , respondents will be asked to self-rate their vegetable intake and physical activity levels. In step 2, their intentions and confidence regarding increasing these amounts over the next 6 months. Participants with moderate and high vegetable intake and physical activity levels will be categorised into action (adopting healthy behaviour within the next 6 months) or maintenance stage (maintaining healthy behaviour for at least 6 months).

Participants who report a low intake of vegetables or participate in little or no physical activity or do not know their vegetable intake or level of physical activity will be categorised into: (a) precontemplation stage (have no intention of changing their behaviour within the next 6 months); (b) contemplation stage (participants who thought of making change in practices within the next 6 months) and (c) preparation stage (respondents planning to change practices within the next 6 months).

\section{Conduct of research}

\section{Questionnaire development}

The questionnaire was designed to measure participants' knowledge and practices around walking and vigorous physical activity, consumption of vegetables, SSB and BMI. The questionnaire was developed in close consultation with the Arabic community and experts in health promotion, research and evaluation, physical activity and nutrition. Questions on knowledge were not validated but pretested on a small sample of Arabic community members. Questions on frequency and duration of walking and vigorous activities and vegetable consumption were adopted from the Active Australia Survey ${ }^{33}$ and New South Wales Population Health Survey. ${ }^{32}{ }^{34}$ These questionnaires have been validated among the Australian population but not necessarily among people of Arabicspeaking background. Questions on Stage of Change in relation to recommended dietary changes were adopted from Chee Yen et al, ${ }^{37}$ pretested and adapted for research into physical activity. The questions on SSBs were adopted from the New South Wales Population Health Survey ${ }^{32} 34$ (see table 3).

\section{Training}

Health Promotion Officers, Multicultural Health Workers (MHWs) and Bilingual Community Educators (BCEs) were trained over 3 days by research and evaluation staff on all aspects of data collection procedures using hardcopy or online survey tools. Three MHWs fluent in both Arabic and English were trained as master interviewers who refined the skills of the BCEs. The training period was 1 week and occurred in three stages:

1. Training of three MHWs as master interviewers.

2. Role play sessions by master interviewers to refine their interview skills.

3. Training of BCEs by master interviewers. This team will administer the baseline and follow-up questionnaires via face to face interviews.

\section{Pilot testing}

Prior to the baseline interviews, the survey questionnaire was field tested by trained interviewers on a small sample of Arabic speakers to determine the appropriateness of the content, its length and cultural sensitivity. Following pretesting, the questionnaire was shortened from 30 to 20 min. Prior to final administration, the online survey will be piloted with a small number of individuals to ensure appropriateness.

\section{Recruitment and selection of participants \\ Survey sample size}

The total sample for this evaluation consists of 1540 adults from two independent samples (preintervention, $\mathrm{n}=770$; postintervention, $\mathrm{n}=770$ ) living in the Bankstown area of NSW. A sample of this size, based on a power of $80 \%$ and alpha of 0.05 , will detect $7 \%$ change (from $37 \%$ to $44 \%$ ) in the estimated level of physical activity among participants. $^{7}$

Participants: inclusion criteria and sources of recruitment

Respondents who meet the inclusion criteria: of Arabicspeaking background; live in Bankstown or surrounding area (places with a high proportion of Middle-Eastern residents) and aged between 18 and 50 years will be eligible to participate.

Participants for the preintervention (baseline) and postintervention surveys will be recruited, applying non-probability sampling methods. The sampling frame includes recruitment of participants from a number of settings. These settings include places such as shopping centres, school and cultural events where adults can be approached to participate. This method of recruitment was chosen because other methods have yielded low response rates, ${ }^{38}$ logistic difficulties and high costs. To increase robustness and overcome limitations in generalising the findings, three measures will be taken: (i) recruitment of an adequate sample for both baseline and postintervention surveys; (ii) recruitment from multiple but similar sources to address sample variations between the baseline and follow-up participants and (iii) ensure our sample reflects the sociodemographic distribution of the Arabic-speaking communities in the Bankstown area.

\section{Quantitative data management and analysis}

Analysis will be performed using the SPSS V.24.0 (SPSS, 2015) for descriptive statistics and statistical significance 
Table 3 Survey instrument-sequence and details of survey items

\begin{tabular}{ll}
\hline Physical activity & Questions \\
\hline Knowledge of physical activity & Two items: How many times per week and how many minutes per week? \\
Walking continuously for $10 \mathrm{~min}$ & Two items: How many times per week and how many minutes per week? \\
Vigorous physical activity & Two items: How many times per week and how many minutes per week?
\end{tabular}

Awareness and use of physical Six items: Are you aware of and do you use any of the following physical activity activity opportunities opportunities in the Bankstown area? Cycle ways, walking tracks, parks, outdoor gyms, walking groups and others.

Physical activity-Stages of Six items: precontemplation/contemplation (no intention); planning (intention) action Change (practising) and maintenance (practising regularly).

Physical activity - perceived barriers/enablers

Four items: the following statements ask your opinion about your physical activity: (multiple response-tick all that apply).

I have the time to do physical activity; I don't have people to do physical activity with; Family responsibilities do not make it difficult to be physically active; It is expensive to do physical activity.

Sedentary behaviour One item: Currently, on average, how many minutes per day do you spend sitting? (eg, watching television, using computer, smart phones, for pleasure).

\section{Nutrition}

Actual vegetable intake

One item: How many serves of vegetables do you usually eat each day? (include fresh, frozen and tinned vegetables and legumes eg, lentils, chickpeas etc). One serve = $1 / 2$ cup cooked or 1 cup of salad vegetables.

Knowledge of recommended One item: How many serves of vegetables do you think you should eat each day? (include vegetables intake fresh, frozen and tinned vegetables and legumes eg, lentils, chickpeas, etc) One serve $=1 / 2$ cup cooked or 1 cup of salad vegetables.

Vegetable intake-Stages of Six items: precontemplation/contemplation (no intention); planning (intention) action Change (practising) and maintenance (practising regularly).

Vegetable intake-perceived One item: the next question asks your opinion about eating enough vegetables.

barriers/enablers

Vegetables are expensive to buy; Preparing vegetables takes a lot of time; I feel confident preparing vegetable dishes; It is more important to eat other foods eg, meat-is this an open ended question or do we give options?

Sugar-sweetened beverage Three items: How many cups of SSB per day; How many cups of fruit juice per day? How many cups of black tea (with sugar) per day?

Body mass index

Two items: self-reported height $(\mathrm{cm})$ and weight $(\mathrm{kg})$

Two items: objectively measured height $(\mathrm{cm})$ and weight $(\mathrm{kg})$

Demographic variables

Gender, age, income, employment status, marital status, education, main language spoken at home, born in Australia, year of migration, use of social media, main type of social media used.

$(\mathrm{p}=0.05)$ tests. Details of planned statistical tests in this study are given below.

Pearson's $\chi^{2}$ test or the Fisher's exact test to assess associations between sociodemographic characteristics, knowledge levels, intention to change, social cognitive factors and intake of vegetables (adequate/inadequate), levels of physical activity (adequate/inadequate) and SSB consumption (0 SSB/day, occasional SSB, $\geq 1 /$ day).

Unpaired t-test to compare the mean differences between preintervention and postintervention data for: (a) knowledge of recommended physical activity levels and vegetable intake, (b) amount of physical activity (walking and vigorous activity), (c) serves of vegetable intake per day and (d) volume of SSBs per day. The paired t-test test will be used to assess the differences between self-reported and measured BMIs. One way analysis of variance (ANOVA) and factorial (two-way)
ANOVA will be used to test for significant differences (using Tukey's HSD test) in the mean of outcome variables by sociodemographic characteristics. Non-parametric tests will be used as appropriate. To examine the relationship between predictor variables and dependent variables, binary or linear regression tests will be conducted. Univariate regression will be initially used to examine the relationship between predictor and dependent variables. Significant variables from the univariate regression models will then be entered into a multivariable regression model, and the backward selection will be used to derive the final model. Associations will be presented as adjusted ORs with 95\% CIs. We will adjust for baseline lifestyle variables, such as BMI, physical activity and SSB-consumption levels and key sociodemographic variables such as age, gender and education levels, in the regression models. 


\section{Qualitative component}

\section{Evaluation design}

A qualitative mixed-methods approach will be used to identify community needs and priorities, and map intervention implementation strategies and solutions. Such an approach will also enable the study's authors to explore participants' perceptions of whether, and to what extent, AHWP activities have had an impact on their behaviour, information that can be used to test the quantitative objectives of the study. Qualitative data will be collected using focus group discussions and in-depth interviews at three different stages (table 2) which includes: (i) formative evaluation (2015-2016); (ii) process evaluation of 'Eat-Move-Live Healthy Programme' (2018-2019) and (iii) follow-up evaluation of 'Eat-Move-Live Healthy Programme' (2020-2021) (see table 2 for outcome measures.

\section{Participant selection}

The initial sample for each of these phases will include up to seven focus groups (with 6-8 participants) and 10 in-depth interviews (table 2). However, whether to conduct more interviews and focus group discussions will be determined by our findings from initial data analysis that provides clues to data saturation. Participants in our focus group discussions and in-depth interviews will be recruited using purposive sampling methods from multiple sources, such as members of key partner organisations, AHWP programme participants and programme coordinators for conducting interviews and focus group discussions to achieve variation in perceptions related to the impact of AHWP activities.

\section{Data collection procedures}

Pretesting and conducting interviews and focus group discussions

The project team will develop multiple open-ended topic guides for conducting focus groups and interviews. The guides will be further refined after expert review, pretested on a sample of project participants and modified where required. Pretesting will assess the appropriacy and scope of interview questions and the duration of group discussions and interviews.

Face-to-face interviews and focus group discussions will be conducted by trained staff. One of the project members will contact the eligible participants by telephone before their scheduled appointment to explain the study, obtain their consent and set an appointment with them. The focus groups and interviews will last for approximately $60 \mathrm{~min}$.

\section{Patient and public involvement}

All information obtained in connection with this study that can identify a participant will remain confidential. The project steering committee members will have the opportunity to review the report and provide their feedback prior to publication. The data will be de-identified prior to analysis, and findings will be reported in a way that individual participants will not be identifiable.

\section{Qualitative data management and analysis}

Data from focus group discussions and interviews will be electronically recorded, professionally transcribed. NVivo V.10 (QSR International) will be used to organise and manage the data. A combination of deductive and inductive methods of data analysis will be conducted. The deductive analysis will use the Anderson model of health utilisation and SCT, while the inductive method will follow the Grounded Theory.

The first stage of analysis will develop a coding scheme consisting of themes, subthemes and their definitions. In the second stage, researchers will negotiate relationships within and between the themes, subthemes and demographic information. In cases of disagreement, a solution will be found by clarifying and discussing the findings until mutual agreement is reached. The data gathered from the focus groups and individual interviews will be compared with capture qualitative dimensions of the data. For example, focus group and interview participants' responses on specific beliefs and practices in relation to physical activity and dietary intake will be triangulated with findings obtained from survey respondents. Finally, findings will be presented to team and steering committee members from various organisations working with Arabic-speaking communities to validate interpretation.

\section{Ethics and dissemination}

The confidentiality of participants and information collected from them will be protected and remain unidentifiable in the dissemination of findings. To ensure that the findings of this project are useful and accessible, we will, in conjunction with our stakeholders, disseminate our findings to a range of audiences. Our results will be summarised in lay language (in both Arabic and English) to ensure continuous engagement and partnership with people of diverse backgrounds (community members, partner organisations, health services and health professionals). We also aim to publish in peer-reviewed journals and also share our findings with colleagues at academic conferences. These initiatives will contribute to an evidence base that can be used to guide and inform the implementation of a multistrategy community-based intervention among adults of Arabic-speaking background and may also have relevance for other culturally diverse communities.

\section{DISCUSSION}

To the best of our knowledge, this is the first intervention using a comprehensive, multistrategic, multilevel community-based intervention to address low levels of physical activity, insufficient intake of vegetables and high SSB intake in the Arabic-speaking community in Australia. This study focuses on major risk factors for chronic non-communicable diseases, especially behaviours such as physical inactivity and unhealthy diet. The aim of this study is to determine whether the implementation and effectiveness 
of a multistrategy intervention can cause participants to increase levels of physical activity and vegetable intake and reduce mean SSB consumption by $7 \%$. The study's findings have the potential to enhance the knowledge and self-efficacy of Arabic-speaking community members in respect of healthy living. For example, factors identified as contributing (both positively and negatively) to lifestyle habits among the population could be strategically tailored in enhancing promotion of healthy living in a culturally sensitive manner.

\section{Current study status and timelines}

The data collection in this study will be carried out across successive stages (baseline data: 2016-2018, process evaluation: 2019-2020 and post evaluation: 2019-2021).

Acknowledgements We acknowledge participants for their time and effort to participate in this study and Francis Fox for his support in editing this paper.

Contributors AHWP investigators, DW, PA, JR, VK: were responsible along with KW and MW for identifying the research question, the design of the protocol and obtaining ethics committee approval. DW, JR, others: were responsible for recruiting study participants for all phases of this study. VK, PA: were responsible for data management and analysis. BJ: provided technical inputs for AHWP and the development of this manuscript. VK: wrote the manuscript; all authors contributed towards the critical review and approval of the final version.

Funding This work is internally supported by Health Promotion Service, South Western Sydney Local Health District.

Competing interests None declared.

Patient consent for publication Obtained.

Ethics approval The study protocol was approved by the Human Research Ethics Committee of SWSLHD (HREC/16/LPOOL/303).

Provenance and peer review Not commissioned; externally peer reviewed.

Open access This is an open access article distributed in accordance with the Creative Commons Attribution Non Commercial (CC BY-NC 4.0) license, which permits others to distribute, remix, adapt, build upon this work non-commercially, and license their derivative works on different terms, provided the original work is properly cited, appropriate credit is given, any changes made indicated, and the use is non-commercial. See: http://creativecommons.org/licenses/by-nc/4.0/.

\section{REFERENCES}

1. Australian Bureau of Census CNSw. New South wales: Census, 2016. http://abs.gov.au/ausstats/abs@.nsf/mediareleasesbyRelea seDate/3DBA1B3973A0286ACA258148000D0DB8?OpenDocument

2. NSW Health. South Western Sydney Local Health District. Health Profile of Local Communities: 2014. https://www.swslhd.health.nsw. gov.au/planning/content/pdf/SWSLHD\%20Community\%20Profile\% 20Summary\%20070814.pdf

3. Hardy LL, Hector D, Saleh S, et al. Australian Middle Eastern parents' perceptions and practices of children's weight-related behaviours: talking with Parents' Study. Health Soc Care Community 2016;24:e63-71.

4. Centre for Epidemiology and Research. 2006-2009 Report on Adult Health by Country of Birth from the New South Wales Population Health Survey. Sydney: NSW Department of Health, 2010.

5. Pate RR, Pratt M, Blair SN, et al. Physical activity and public health. A recommendation from the Centers for Disease Control and Prevention and the American College of Sports Medicine. JAMA 1995;273:402-7.

6. Lee IM, Shiroma EJ, Lobelo F, et al. Effect of physical inactivity on major non-communicable diseases worldwide: an analysis of burden of disease and life expectancy. Lancet 2012;380:219-29.

7. NSW Governament Health. NSW Healthy Eating and Active Living Strategy: Preventing overweight and obesity in New South Wales 2013-2018, 2013:22.

8. Caperchione CM, Kolt GS, Tennent R, et al. Physical activity behaviours of Culturally and Linguistically Diverse (CALD) women living in Australia: a qualitative study of socio-cultural influences. BMC Public Health 2011;11:26.

9. Caperchione CM, Kolt GS, Mummery WK. Physical activity in culturally and linguistically diverse migrant groups to Western society: a review of barriers, enablers and experiences. Sports Med 2009;39:167-77.

10. Caperchione CM, Kolt GS, Mummery WK. Examining physical activity service provision to culturally and linguistically diverse (CALD) communities in Australia: a qualitative evaluation. PLoS One 2013;8:e62777.

11. Project AHW. Findings from community consultations (formative evaluation) of Arabic Healthy Weight Project. 2016

12. Hector D, King L, Hardy L, et al. Evidence update on obesity prevention; Across the life-course Prepared for NSW Ministry of Health. Sydney: Physical Activity Nutrition Obesity Research Group, 2012. http://sydney.edu.au/medicine/public-health/ prevention-research/news/reports/Evidence\%20update\%20life \% 20course.pdf

13. Kite JHD, St George A, Pedisic Z, et al. Comprehensive sector-wide strategies to prevent and control obesity: what are the potential health and broader societal benefits? A case study from Australia. 2015.

14. Sulaiman ND, Furler JS, Hadj EJ, et al. Stress, culture and 'home': social context in Turkish and Arabic-speaking Australians' views of diabetes prevention. Health Promot J Austr 2007;18:63-8.

15. Renzaho AM, Mellor D, Boulton K, et al. Effectiveness of prevention programmes for obesity and chronic diseases among immigrants to developed countries - a systematic review. Public Health Nutr 2010;13:438-50.

16. CEH (Centre for Cultural Ethnicity and Health). Engaging Culturally and Linguistically Diverse Communities in Physical Activity: A Discussion Paper. Melbourne, Victoria: CEH, 2006.

17. QLD Health (Queensland Health). Engaging culturally and linguistically diverse (CALD) Queenslanders in physical activity: Findings of the CALD Physical Activity Mapping Project (2010). Brisbane: Division of the Chief Health Officer, Queensland Health, 2010.

18. Lee SM. Physical activity among minority populations: what health promotion practitioners should know-a commentary. Health Promot Pract 2005;6:447-52.

19. Wakefield MA, Loken B, Hornik RC. Use of mass media campaigns to change health behaviour. Lancet 2010;376:1261-71.

20. Perusco A, Poder N, Mohsin M, et al. Guirguis Ma'feesh cigara men gheir khosara: Project Report. Liverpool, Australia: South Western Sydney Area Health Service, 2011.

21. Kent J, Thompson SM, Jalaludin B. Healthy Built Environments: a review of the literature. Sydney: Healthy Built Environments Program, City Futures Research Centre, UNSW, 2011.

22. Clarke R, Armstrong R, Waters E. Local government and obesity prevention: An evidence resource. Interventions to prevent obesity in early year settings; tackling food insecurity and built environment changes to support physical activity: CO-OPs Secretariat, Deakin University, Geelong, 2010.

23. Health Statistics, NSW. Overweight and Obesity in adults Physical activity in adults by country of birth group, persons aged 16 years and over, NSW, 2017. http://www.healthstats.nsw.gov.au/Indicator/ beh_phys_age/beh_phys_cob_snap

24. Bauman AE. Don, Nutbeam Evaluation in a nutshell: practical guide to the evaluation of health promotion programs. Australia: McGrawHill Education, 2014.

25. Scheme NDS. Understanding pre-diabetes. 2016. Available: https:// www.ndss.com.au/understanding-pre-diabetes [Accessed 8 May 2018].

26. Brown WJ BA, Bull FC, Burton NW. Development of Evidence-based Physical Activity Recommendations for Adults (18-64 years): Report prepared for the Australian Government Department of Health, 2012.

27. Vartanian LR, Schwartz MB, Brownell KD. Effects of soft drink consumption on nutrition and health: a systematic review and metaanalysis. Am J Public Health 2007;97:667-75.

28. Blekkenhorst LC, Sim M, Bondonno CP, et al. Cardiovascular health benefits of specific vegetable types: a narrative review. Nutrients 2018;10:595.

29. Chau J, Smith BJ, Bauman A, et al. Recent trends in physical activity in New South Wales. Is the tide of inactivity turning? Aust N Z J Public Health 2008;32:82-5.

30. Rose G, Day S. The population mean predicts the number of deviant individuals. BMJ 1990;301:1031-4.

31. Meleis Al, Jonsen AR. Ethical crises and cultural differences. West $J$ Med 1983;138:889-93.

32. Centre for Epidemiology and Evidence NMoH. NSW Population Health Survey 2014. 
33. Australian Institute of Health and Welfare (AlHW). The Active Australia Survey: a guide and manual for implementation aar. Canberra: AlHW, 2003.

34. AGDH (Australian Government Department of Health). Australia's Physical Activity and Sedentary Behaviour Guidelines. www.health. gov.au/internet/main/publishing.nsf/content/health-pubhlth-strategphys-act-guidelines

35. World Health Organisation. Obesity and Overweight fact sheet. 2016. http://www.who.int/mediacentre/factsheets/fs311/en/Obesity
36. Australian Governament N, Department of Health and aging. Eat for Health - Australian Dietary Gudelines (summary). 2013.

37. Chee Yen W, Mohd Shariff Z, Kandiah M, et al. Stages of change to increase fruit and vegetable intake and its relationships with fruit and vegetable intake and related psychosocial factors. Nutr Res Pract 2014;8:297-303.

38. Payne WR, Harvey JT, Dharmage SC. Immigrant Physical Activity Study. A Report to The Victorian Health Promotion Foundation. 2011. 